BMJ Open

Diabetes

Research

\& Care

\section{Glycated hemoglobin (HbA1c) as diagnostic criteria for diabetes: the optimal cut-off points values for the Pakistani population; a study from second National Diabetes Survey of Pakistan (NDSP) 2016-2017}

To cite: Basit A, Fawwad A, Abdul Basit K, et al. Glycated hemoglobin (HbA1c) as diagnostic criteria for diabetes: the optimal cutoff points values for the Pakistani population; a study from second National Diabetes Survey of Pakistan (NDSP) 2016-2017.

BMJ Open Diab Res Care 2020;8:e001058. doi:10.1136/ bmjdrc-2019-001058

Received 18 November 2019 Revised 1 February 2020 Accepted 24 March 2020

Check for updates

C Author(s) (or their employer(s)) 2020. Re-use permitted under CC BY-NC. No commercial re-use. See rights and permissions. Published by BMJ.

For numbered affiliations see end of article.

Correspondence to Dr Abdul Basit; abdulbasit@bide.edu.pk

\section{ABSTRACT}

Aim Glycated hemoglobin ( $\mathrm{HbA1c}$ ) cut-off values as diagnostic tool in diabetes and prediabetes with its concordance to oral glucose tolerance test (OGTT) in Pakistani population.

Methodology Data for this substudy was obtained from second National Diabetes Survey of Pakistan (NDSP) 2016-2017. With this survey, 10834 individuals were recruited and after excluding known subjects with diabetes, 6836 participants fulfilled inclusion criteria for this study. Demographic, anthropometric and biochemical parameters were obtained. OGTT was used as standard diagnostic tool to screen population and $\mathrm{HbA} 1 \mathrm{c}$ for optimal cut-off values. Participants were categorized into normal glucose tolerance (NGT), newly diagnosed diabetes (NDD) and prediabetes.

Results Out of 6836 participants, 4690 (68.6\%) had NGT, 1333 (19.5\%) had prediabetes and $813(11.9 \%)$ had NDD by OGTT criteria with median (IQR) age of 40 (31-50) years. Optimal HbA1c cut-off point for identification of diabetes and prediabetes was observed as 5.7\% ((AUC $(95 \% \mathrm{Cl})=0.776(0.757$ to 0.795$), \mathrm{p}<0.0001))$ and $5.1 \%$ $((\operatorname{AUC}(95 \% \mathrm{Cl})=0.607$ (0.590 to 0.624$), \mathrm{p}<0.0001))$, respectively. However, out of $68.6 \%$ NGT subjects identified through OGTT, $24.1 \%$ and $9.3 \%$ participants were found to have prediabetes and NDD, respectively by using $\mathrm{HbA} 1 \mathrm{c}$ criteria. By using both $0 \mathrm{GTT}$ and $\mathrm{HbA} 1 \mathrm{C}$ criteria, only $7.9 \%$ and $7.3 \%$ were observed as prediabetes and diabetes, respectively.

Conclusion Findings from second NDSP demonstrated disagreement between findings of $0 \mathrm{GTT}$ and $\mathrm{HbA} 1 \mathrm{c}$ as diagnostic tool for Pakistani population. As compared with international guidelines, $\mathrm{HbA} 1 \mathrm{c}$ threshold for prediabetes and NDD were lower in this part of world. HbA1c as diagnostic tool might require ethnic or regional-based modification in cut-off points, validated by relevant community-based epidemiological surveys.

\section{INTRODUCTION}

Globally, type 2 diabetes is a burgeoning public health challenge and a significant

\section{Significance of this study}

What is already known about this subject?

- HbA1c offers some advantages over fasting glucose and oral glucose tolerance test (OGTT). Racial and ethnic variations are observed as diagnostic tool for diabetes.

What are the new findings?

- The findings from second NDSP demonstrated the disagreement between the findings of OGTT and $\mathrm{HbA1C}$ as diagnostic tool for the Pakistani population. As compared to International guidelines, $\mathrm{HbA} 1 \mathrm{C}$ threshold for prediabetes and NDD were found to be lower in this part of the world.

- $\mathrm{HbA1C}$ as a diagnostic tool might require ethnic or regional-based modification in cut-off points, validated by relevant community based epidemiological surveys.

How might these results change the focus of research or clinical practice?

- $\mathrm{HbA1C}$ as a diagnostic tool has its limitations. Local and regional guidelines should place $\mathrm{HbA1C}$ as diagnostic and prognostic tool in accordance with the available publish data.

contributor to non-communicable diseaserelated morbidity and mortality, particularly in resource-constrained countries. ${ }^{2}$ In Pakistan, recent second National Diabetes Survey of Pakistan (2nd NDSP) 2016-2017 has estimated the overall prevalence of diabetes to be $26.3 \%$. $^{3}$

Oral glucose tolerance test (OGTT) has been considered as the standardized diagnostic criteria for the classification of diabetes and prediabetes for decades. ${ }^{45}$ The uncertainty of fasting phase, poor concordance of 
fasting plasma glucose (FPG), 2-hour glucose tolerance (2hGT) values, day-to-day high glucose fluctuations and an alternative to glucose measurements for the diagnosis of diabetes has long been sought. ${ }^{6} 7$ Performance of HbA1c with OGTT for diagnosing diabetes and prediabetes has been compared in many studies. ${ }^{8}$ Racial and ethnic variations in $\mathrm{HbAlc}$ values were also observed for the same degree of hyperglycemia affecting its utilization as a modality for diagnosing diabetes. ${ }^{19} \mathrm{HbAlc}$ offers some advantages over fasting glucose and 2hGT (OGTT) tests, as it can be performed at any time of the day with no requirement for overnight fasting and glucose challenge. The results do not get affected by delayed analysis after collection if properly stored as per the standard guidelines. ${ }^{1} 10$

Studies have symbolized the evidence that convenience of sampling, unaffected outcome with dietary intake or compliance with fasting and stabilized analytical concentration in blood make HbAlc as a useful screening tool for diabetes in population and its complications, however, it is still not a validated tool for new diagnosis of diabetes in low-income and middle-income world. ${ }^{11} 12$ The common underlying causes for underestimation of HbAlc due to either hemoglobinopathies, hemolysis, hypertriglyceridemia, drugs and vitamin supplementations further raises the concern for diagnosing diabetes at $6.5 \%(48 \mathrm{mmol} / \mathrm{mol}) .{ }^{13-16}$

It, therefore, seems imperative to determine the regional optimal cut-off values for $\mathrm{HbAlc}$ in order to have a useful, convenient and reliable tool for identifying the subjects with prediabetes and diabetes. Hence, this study aims to evaluate HbA1c cut-offs as a diagnostic tool in diabetes and prediabetes with its concordance to the OGTT in Pakistani population.

\section{METHODOLOGY}

Data for this substudy was obtained from 2nd NDSP (2016-2017). ${ }^{3}$ Duration of the survey was 19 months from February 2016 to August 2017. Informed consent was obtained from each participant. ${ }^{3}$

The main objective of the survey was to assess the prevalence of type 2 diabetes and its associated risk factors in urban and rural areas of all four provinces of Pakistan that includes Punjab, Sindh, Khyber Pakhtunkhwa (KPK) and Baluchistan (as defined in the latest available census). The estimated sample size was 10800 . Known and unknown diabetics, hypertensives and with other cardiometabolic risk factors were included as per designed methodology. Sample was taken from each province (stratum) with respect to its population size. In each province, tehsils or towns as subclusters were selected from identified clusters using probability proportional to size technique as defined in 2nd NDSP (2016-2017). Identified clusters were selected using the 'rule of thumb': number of clusters $(\mathrm{k})=(\text { sample size of stratum/2 })^{0.5}$. Total of 21 (tehsils/towns) from urban and 23 (tehsils/towns) from rural areas were selected for the survey. Households were identified and selected members of every 10th household were advised to come to the camp after an overnight fast. Probability sampling and multistage stratified sampling technique were used to calculate the sample size. ${ }^{3}$

A predesigned questionnaire comprising detailed demographic, medical, social and obstetric history was filled by trained medical and research officers via a detailed interactive session. Self-questionnaire filling was not allowed. Unanswered or indecisive responses were considered as negative. Blood samples were drawn for the assessment of biochemical parameters. The clinical and anthropometric measurements included height, weight, blood pressure, waist circumference and waistto-hip ratio, while biochemical parameters were OGTT, HbA1c and fasting lipid. Specifications of equipment was uniform throughout the study for standardization and to ensure quality control and sample were run as per standardized protocol. ${ }^{317}$ In the survey, OGTT was used as a standard diagnostic test to screen the population. In this substudy, HbA1c using the American Diabetes Association (ADA) criteria was also evaluated in the diagnosis of prediabetes and diabetes with its optimal cut-off values. To determine and differentiate between the optimal HbAlc cut-offs for Pakistani population and its concordance with OGTT, all those subjects who had known type 2 diabetes or were on antidiabetes treatment, and subjects missing their HbA1c levels were excluded from this substudy.

In the 2nd NDSP (2016-2017), 10834 individuals were recruited for diabetes, out of which 6836 participants fulfilling inclusion criteria were included (participants with known diabetes and who have missing HbAlc were excluded from this substudy). ${ }^{3}$ Participants were categorized as normal glucose tolerance (NGT), newly diagnosed diabetes (NDD) and prediabetes. Known diabetes was considered if the subject had been diagnosed as having diabetes by a physician or definition by WHO. ${ }^{5} 18$ Diabetes and prediabetes (intermediate hyperglycemia) were diagnosed according to WHO categorization as done in the 2nd NDSP (2016-2017). ${ }^{3}$ As per WHO definition, results of plasma glucose testing were categorized as follows: isolated impaired fasting glucose (IFG) - FPG level between 110 and $125 \mathrm{mg} / \mathrm{dL}$ with 2-hour postglucose level (PGL) $\leq 140 \mathrm{mg} / \mathrm{dL}$. Isolated impaired glucose tolerance-fasting glucose level $<110 \mathrm{mg} / \mathrm{dL}$ and 2-hour PGL between 141 and $199 \mathrm{mg} / \mathrm{dL}$. Newly diagnosed diabetes was defined as FPG level $\geq 126 \mathrm{mg} / \mathrm{dL}$ or 2-hour PGL $\geq 200 \mathrm{mg} / \mathrm{dL}$ or both. For the diagnosis of diabetes using $\mathrm{HbAlc}$ as a diagnostic tool, the ADA standards of care were used. $\mathrm{HbAlc} \geq 6.5 \%(48 \mathrm{mmol} / \mathrm{mol})$ was diagnosed as diabetes, while HbAlc between $5.7 \%$ and $6.4 \%$ (39 and $46 \mathrm{mmol} / \mathrm{mol}$ ) was considered as prediabetes with results of available OGTT. ${ }^{3519}$

Height, body weight, waist circumference and blood pressure were measured using standardized techniques. Body mass index (BMI) was calculated as weight in kilograms divided by height in meter squared $(\mathrm{kg} /$ $\mathrm{m}^{2}$ ). Blood pressure was measured with a mercury 
sphygmomanometer. Plasma glucose was measured using the glucose oxidase peroxidase method, total cholesterol by cholesterol oxidase phenol 4-amino antipyrine peroxidase (CHOD-PAP) method, triglycerides by glycerol phosphate oxidase-p-amino phenazone method, high-density lipoprotein cholesterol by homogeneous enzymatic calorimetric method, low-density lipoprotein cholesterol by CHOD-PAP method and HbAlc by highperformance liquid chromatography method as reported earlier in 2nd NDSP (2016-2017). ${ }^{3}$

\section{Statistical analysis}

Statistical analysis was performed using Statistical Package for Social Sciences V.20. Variables were expressed as median (IQR) or proportions. Kruskal-Wallis H nonparametric test was used to compare continuous variables and $\chi^{2}$ test for categorical variables. With OGTT test as the gold standard, receiver operating characteristic curve (ROC) was used for the multiclass variable to define area under the curve (AUC) of HbAlc for diagnosing diabetes and prediabetes. ROC curve was used with OGTT (categorical variable) as state variable and $\mathrm{HbAlc}$ (continuous variable) as test variable. The cut-off for HbAlc was noted where sensitivity and specificity were optimal. Data were divided with respect to age and gender for both diabetics and prediabetics. The optimal HbA1c cut-off value was selected where sum of sensitivity and specificity was maximum. Statistical significance was determined as a two-sided $\mathrm{p}<0.05$.

\section{RESULTS}

OGTT was performed for 10834 individuals, however, only 6836 participants fulfilled the inclusion criteria for this substudy. Out of 6836 participants, $4690(68.6 \%)$ had NGT, 1333 (19.5\%) had prediabetes and 813 (11.9\%) had NDD by OGTT criteria.

Table 1 shows the characteristics of subjects by glucose status according to OGTT in all three groups. Female participants were dominant $3952(57.8 \%)$ as compared with males $2884(42.2 \%)$. The median (IQR) age of participants was 40 (31-50) years. Positive family history of diabetes mellitus was 827 (22.2\%) in NGT group, 353 $(30.0 \%)$ in prediabetes and $183(28.8 \%)$ in NDD group. Significantly higher results were observed for baseline and biochemical parameters in prediabetes except for primary education or more and HDL as compared with participants with NGT, while in NDD, this trend was observed for all variables except for LDL as compared with NGT group.

Table 2 is showing the optimal HbAlc cut -off point for identification of diabetes as $5.7 \%(39 \mathrm{mmol} / \mathrm{mol})$ ( $($ AUC $(95 \% \mathrm{CI})=0.776(0.757$ to 0.795$), \mathrm{p}<0.0001))$ with a sensitivity and specificity of $59.5 \%$ and $85.9 \%$, respectively. The sensitivity and specificity of $\mathrm{HbA1c}$ value of $6.5 \%(48 \mathrm{mmol} / \mathrm{mol})$ (recommended by ADA) to classify diabetes were $43.40 \%$ and $92.60 \%$, respectively. ${ }^{5}$ The optimal HbA1c cut-off point for identification of prediabetes was $5.1 \%(32 \mathrm{mmol} / \mathrm{mol}) \quad((\mathrm{AUC}(95 \% \mathrm{CI})=0.607$

\begin{tabular}{|c|c|c|c|c|}
\hline Variable & Overall & Normal & Prediabetes & NDD \\
\hline $\mathrm{N}$ & 6836 & 4690 (68.6\%) & 1333 (19.5\%) & 813 (11.9\%) \\
\hline Age (years) & $40(31-50)$ & $39(29-49)$ & $45(35-55)^{\star \star \star}$ & $49(40-58)^{\star \star \star}$ \\
\hline \multicolumn{5}{|l|}{ Gender } \\
\hline Male & $2884(42.2 \%)$ & 1963 (41.9\%) & $562(42.2 \%)$ & $359(44.2 \%)$ \\
\hline Female & $3952(57.8 \%)$ & $2727(58.1 \%)$ & $771(57.8 \%)$ & $454(55.8 \%)$ \\
\hline Positive family history of DM & $1363(24.6 \%)$ & $827(22.2 \%)$ & $353(30.0 \%)^{\star \star \star}$ & $183(28.8 \%)^{\star \star \star}$ \\
\hline Primary education or more & 3045 (53.3\%) & $2132(54.3 \%)$ & $604(52.2 \%)$ & $309(49.1 \%)^{*}$ \\
\hline Tobacco & $814(14.1 \%)$ & $508(12.8 \%)$ & $199(16.9 \%)^{\star \star \star}$ & $107(16.7 \%)^{\star \star}$ \\
\hline Body mass index $\left(\mathrm{kg} / \mathrm{m}^{2}\right)$ & $26.3(22.8-30.3)$ & $26.2(22.5-30.1)$ & $26.5(23.3-30.4)^{*}$ & $27.1(23.7-30.7)^{\star \star}$ \\
\hline Waist circumference (cm) & $91(81-99)$ & $89(81-98)$ & $92(86-102)^{\star \star \star}$ & $94(86-103)^{\star \star \star}$ \\
\hline Systolic blood pressure (mm Hg) & $120(110-130)$ & $120(110-130)$ & $120(115-140)^{\star \star \star}$ & $130(120-140)^{\star \star \star}$ \\
\hline Diastolic blood pressure $(\mathrm{mm} \mathrm{Hg})$ & $80(71-90)$ & $80(70-90)$ & $80(80-90)^{\star \star \star}$ & $80(80-90)^{\star \star \star}$ \\
\hline Total cholesterol (mg/dL) & $185(151-222)$ & $183(149-218)$ & $188(157-227)^{\star \star}$ & $192(154-230)^{\star \star}$ \\
\hline Triglyceride (mg/dL) & $146(102-218)$ & $138(97-206)$ & $155(108-226)^{\star \star \star}$ & $183(123-269)^{\star \star \star}$ \\
\hline High-density lipoprotein (mg/dL) & $31(21.9-40)$ & $31.6(22.4-40.1)$ & $30.4(22.0-39.7)$ & $29.0(20.1-37.1)^{\star \star \star}$ \\
\hline Low-density lipoprotein (mg/dL) & $118.9(95.1-145.6)$ & $117.3(95-143.4)$ & $122(97-150.4)^{\star \star \star}$ & $121.5(94.2-149.8)$ \\
\hline $\mathrm{HbA1c}(\%)$ & $5.1(4.8-5.5)$ & $5.0(4.8-5.3)$ & $5.2(4.9-5.6)^{\star \star \star}$ & $6.2(5.3-8.4)^{\star \star \star}$ \\
\hline $\mathrm{HbA1c}(\mathrm{mmol} / \mathrm{mol})$ & $32(29-37)$ & $31(29-34)$ & $33(30-38)^{\star \star \star}$ & $44(34-68)^{\star \star \star}$ \\
\hline
\end{tabular}

Data presented as median (IQR) or $\mathrm{n}(\%)$

${ }^{*} \mathrm{P}<0.05,{ }^{* *} \mathrm{p}<0.001,{ }^{* * *} \mathrm{p}<0.0001$.

$\mathrm{DM}$, diabetes mellitus; HbA1c, glycated hemoglobin ; NDD, newly diagnosed diabetes; OGTT, oral glucose tolerance test. 
Table 2 Sensitivity and specificity of different HbA1c cutoff value for diagnosing diabetes and prediabetes

\begin{tabular}{|c|c|c|c|}
\hline Status & $\begin{array}{l}\text { HbA1c \% } \\
\text { (mmol/mol) }\end{array}$ & $\begin{array}{l}\text { Sensitivity } \\
(\%)\end{array}$ & $\begin{array}{l}\text { Specificity } \\
(\%)\end{array}$ \\
\hline \multirow[t]{15}{*}{ Diabetes } & $5.1(32)$ & 79.0 & 57.7 \\
\hline & $5.2(33)$ & 75.4 & 64.8 \\
\hline & $5.3(34)$ & 72.3 & 71.3 \\
\hline & $5.4(36)$ & 67.7 & 76.1 \\
\hline & $5.5(37)$ & 63.8 & 79.9 \\
\hline & $5.6(38)$ & 61.0 & 84.1 \\
\hline & 5.7 (39) & 59.5 & 85.9 \\
\hline & $5.8(40)$ & 57.1 & 87.7 \\
\hline & $5.9(41)$ & 54.2 & 89.1 \\
\hline & $6.0(42)$ & 52.8 & 90.1 \\
\hline & $6.1(43)$ & 51.0 & 90.7 \\
\hline & $6.2(44)$ & 47.6 & 91.6 \\
\hline & $6.3(45)$ & 46.7 & 91.9 \\
\hline & $6.4(46)$ & 45.0 & 92.3 \\
\hline & $6.5(47)$ & 43.4 & 92.6 \\
\hline \multirow[t]{11}{*}{ Prediabetes } & 4.7 (28) & 96.1 & 23.4 \\
\hline & $4.8(29)$ & 80.3 & 30.6 \\
\hline & $4.9(30)$ & 73.9 & 38.7 \\
\hline & $5(31)$ & 64.6 & 51.2 \\
\hline & $5.1(32)$ & 56.9 & 59.7 \\
\hline & $5.2(33)$ & 50.8 & 65.22 \\
\hline & $5.3(34)$ & 40.7 & 74.7 \\
\hline & $5.4(36)$ & 34.7 & 79.1 \\
\hline & $5.5(37)$ & 30.1 & 82.7 \\
\hline & $5.6(38)$ & 24.1 & 86.4 \\
\hline & 5.7 (39) & 21.6 & 88.0 \\
\hline
\end{tabular}

$\mathrm{HbA1c}$ presented \% (mmol/mol).

Bold values indicate optimal cut-point chosen by Youden index $(\mathrm{J})$ method.

$\mathrm{HbA1c}$, glycated hemoglobin.

$(0.590$ to 0.624$), \mathrm{p}<0.0001))$ with a corresponding sensitivity and specificity of $56.9 \%$ and $59.7 \%$, respectively. The sensitivity and specificity of HbAlc value of $5.7 \%$ (39 $\mathrm{mmol} / \mathrm{mol}$ ) (recommended by ADA) to classify prediabetes were $21.3 \%$ and $88.2 \%$, respectively. ${ }^{5}$ Sensitivity and specificity of other HbAlc cut-off values are displayed in table 2.

Table 3 shows the stratified analysis of HbAlc for diagnosing diabetes and prediabetes. The optimal HbA1c cutoff values for detecting NDD and prediabetes were $5.62 \%$ $(38 \mathrm{mmol} / \mathrm{mol})($ AUC $(95 \% \mathrm{CI})=0.776(0.748$ to 0.804$))$ and $5.20 \%(33 \mathrm{mmol} / \mathrm{mol})($ AUC $(95 \% \mathrm{CI})=0.612(0.585$ to 0.638 ) in males and $5.80 \%(40 \mathrm{mmol} / \mathrm{mol}$ ) (AUC $(95 \% \mathrm{CI})=0.777(0.752$ to 0.802$))$ and $5.11 \%(32 \mathrm{mmol} /$ mol) $($ AUC $(95 \% \mathrm{CI})=0.604$ (0.581 to 0.626$))$ in females, respectively. Age-stratified analysis of HbAlc shows the optimal HbAlc cut-off point for diabetes as $5.11 \%$ (32 $\mathrm{mmol} / \mathrm{mol})$ and prediabetes as $4.79 \%(29 \mathrm{mmol} / \mathrm{mol})$ for youth (20-29 years). This trend was $5.94 \%$ (41 mmol/ $\mathrm{mol})$ and $5.16 \%(33 \mathrm{mmol} / \mathrm{mol})$ in $30-39$ years, $5.80 \%$ (40 $\mathrm{mmol} / \mathrm{mol})$ and $5.30 \%(34 \mathrm{mmol} / \mathrm{mol})$ in $40-49$ years, $5.85 \%(40 \mathrm{mmol} / \mathrm{mol})$ and $5.25 \%(34 \mathrm{mmol} / \mathrm{mol})$ in $50-59$ years and $5.67 \%(38 \mathrm{mmol} / \mathrm{mol})$ and $5.11 \%(32$ $\mathrm{mmol} / \mathrm{mol}$ ) in $\geq 60$ years for diabetes and prediabetes, respectively.

Table 4 presents the frequency of NDD and prediabetes by HbA1c and OGTT standard. Among 4690 $(100 \%)$ subjects with NGT by OGTT, $1647(35.1 \%)$ and $636(13.6 \%)$ were identified as prediabetes and NDD, respectively, by HbA1c criteria and only 2407 (51.3\%) were observed with NGT by both criteria. However, out of $1333(100 \%)$ participants with prediabetes diagnosed by OGTT, $474(35.6 \%)$ and $319(23.9 \%)$ were identified with NGT and diabetes by HbA1c criteria, respectively, and $540(40.5 \%)$ were identified prediabetes by both criteria. On the other hand, a total of $813(100 \%)$ subjects were diagnosed with diabetes by OGTT, of whom $135(16.6 \%)$ and $182(22.4 \%)$ were identified with NGT and prediabetes by HbA1c criteria and only 496 (61.0) with NDD by both criteria.

In table 5 , the group by OGTT not HbA1c was compared with group by HbA1c not OGTT. By ADA criteria for HbAlc cut-off values, 955 (13.9\%) and 1829 (26.7\%) participants were detected as NDD and prediabetes, respectively. While $317(4.6 \%)$ and $793(11.6 \%)$ were diagnosed as NDD and prediabetes by OGTT cutoff values, respectively. Only $496(7.3 \%)$ were diagnosed as NDD and $540(7.9 \%)$ as prediabetes by both (HbAlc and OGTT) criteria. Further analysis demonstrates that in NDD, significant results were observed for age, waist circumference, systolic and diastolic blood pressure, HbAlc, fasting blood sugar and random blood sugar in group by HbAlc not OGTT as compared with group by OGTT not HbA1c. This trend was significant in prediabetes for all variables except for age, gender, primary education or more, systolic blood pressure and LDL in group diagnosed by HbA1c not OGTT as compared with group by OGTT not HbA1c.

\section{DISCUSSION}

In this substudy, all participants compared by both criteria were suggesting that $\mathrm{HbAlc}$ as a screening tool for diabetes and prediabetes in comparison to OGTT has non-similar performance. However, the optimal HbAlc threshold for diagnosing diabetes and prediabetes was found to be lower than ADA criteria for our population. Our results are consistent in general to previous studies, that FPG (OGTT or fasting only) measurement performs better than HbAlc for screening NDD and prediabetes. $^{71213}$ But these results as opposed to other study suggesting that the FPG and HbAlc have similar performance as screening modalities for diabetes in a multi-racial Asian population. ${ }^{20}$ 
Table 3 Stratified analysis of $\mathrm{HbA} 1 \mathrm{c}$ for diagnosing diabetes and prediabetes

Optimal HbA1c \%

(mmol/mol) cut-off point Sensitivity (\%)

Specificity (\%) AUC $(95 \% \mathrm{Cl})$

$P$ value

\begin{tabular}{|c|c|c|c|c|c|}
\hline \multicolumn{6}{|l|}{ Diabetes } \\
\hline \multicolumn{6}{|l|}{ Gender } \\
\hline Male & $5.62(38)$ & 61.6 & 83.8 & 0.776 (0.748 to 0.804$)$ & 0.96 \\
\hline Female & $5.80(40)$ & 57.7 & 88.2 & 0.777 (0.752 to 0.802$)$ & \\
\hline \multicolumn{6}{|c|}{ Age (years) } \\
\hline 20-29 & $5.11(32)$ & 50 & 72.2 & 0.645 (0.567 to 0.723$)$ & 0.025 \\
\hline 30-39 & $5.94(41)$ & 49.5 & 91.3 & 0.747 (0.694 to 0.801$)$ & 0.888 \\
\hline $40-49$ & $5.80(40)$ & 61 & 84.4 & 0.757 (0.717 to 0.797$)$ & 0.87 \\
\hline $50-59$ & $5.85(40)$ & 64.6 & 81.9 & 0.779 (0.739 to 0.818$)$ & 0.388 \\
\hline $60+$ & $5.67(39)$ & 65.7 & 75.1 & 0.752 ( 0.710 to 0.794$)$ & \\
\hline \multicolumn{6}{|c|}{ Prediabetes } \\
\hline \multicolumn{6}{|c|}{ Gender } \\
\hline Male & $5.20(33)$ & 48.6 & 69.4 & 0.612 (0.585 to 0.638$)$ & 0.662 \\
\hline Female & $5.11(32)$ & 58.6 & 57.5 & 0.604 (0.581 to 0.626$)$ & \\
\hline \multicolumn{6}{|c|}{ Age (years) } \\
\hline 20-29 & $4.79(29)$ & 77.7 & 34 & 0.562 (0.516 to 0.608$)$ & 0.676 \\
\hline $30-39$ & $5.16(33)$ & 51.5 & 62.6 & 0.588 (0.552 to 0.624$)$ & 0.682 \\
\hline $40-49$ & $5.3(34 I)$ & 46.4 & 68.7 & 0.568 (0.532 to 0.604$)$ & 0.781 \\
\hline $50-59$ & $5.25(34)$ & 58.2 & 84.9 & 0.581 (0.539 to 0.624$)$ & 0.873 \\
\hline $60+$ & $5.11(32)$ & 69.9 & 87.1 & 0.576 (0.532 to 0.620$)$ & 0.771 \\
\hline
\end{tabular}

$\mathrm{HbA} 1 \mathrm{c}$ presented \% (mmol/mol).

AUC, area under the curve; HbA1c, glycated hemoglobin.

In this study, only $7.9 \%$ and $7.3 \%$ participants were identified as prediabetes and NDD, respectively, by using both OGTT and HbAlc criteria. This trend was higher by using only HbAlc cut-offs of ADA criteria as compared with using only OGTT. HbAlc used in diagnosing or screening diabetes has been debated for many years. The HbAlc $>6.1 \%(43 \mathrm{mmol} / \mathrm{mol})$ was the recommended optimum cut-off point for diabetes in most reviewed studies; however, there is also an argument for population-specific cut-off points as optimum cut-offs vary by ethnic group and population prevalence of diabetes. ${ }^{21} \mathrm{In}$ previous study, HbA1c cut-off point of $5.6 \%$ (38 mmol/ mol) was identified for undiagnosed type 2 diabetes, and used by the Japanese Diabetes Society as a supplementary diagnostic criterion. ${ }^{22}$

Similarly, in our population, the optimal HbA1c cut-off point $5.7 \%(39 \mathrm{mmol} / \mathrm{mol})$ was able to identify people with undiagnosed diabetes and $5.1 \%(32 \mathrm{mmol} / \mathrm{mol})$ had an increased risk for progression to type 2 diabetes with high predictive sensitivity than recommended HbAlc values by ADA. Hence, these cut-offs similar to other reported studies are too high to use for screening diabetes in our populations. ${ }^{523} 24$ The discrepancy between our study and others may be caused by differences in study design, study participants demography and area location. Racial differences may affect optimal HbAlc threshold for diagnosing diabetes, therefore, more and more data are still needed from all over the world. ${ }^{25} 26$

Age-stratified analysis of HbAlc for diagnosing diabetes and prediabetes in our study shows the specific trends that also suggest the age-specific diagnostic threshold should be considered, if HbAlc being recommended as diagnostic standard similar to previous studies. ${ }^{623}$ Studies in Dutch and US population also present the HbAlc

\begin{tabular}{|c|c|c|c|c|}
\hline \multirow[b]{2}{*}{ OGTT } & \multicolumn{3}{|c|}{ HbA1c cut-off point } & \multirow[b]{2}{*}{ Total } \\
\hline & $<5.1 \%(32 \mathrm{mmol} / \mathrm{mol})$ & $5.2 \%-5.6 \%(33-38 \mathrm{mmol} / \mathrm{mol})$ & $\geq 5.7 \%$ ( $\geq 39 \mathrm{mmol} / \mathrm{mol})$ & \\
\hline NGT & 2407 (51.3\%) & 1647 (35.1\%) & $636(13.6 \%)$ & $4690(100 \%)$ \\
\hline Prediabetes & $474(35.6 \%)$ & $540(40.5 \%)$ & $319(23.9 \%)$ & $1333(100 \%)$ \\
\hline NDD & 135 (16.6\%) & $182(22.4 \%)$ & 496 (61.0\%) & $813(100 \%)$ \\
\hline
\end{tabular}

HbA1c, glycated hemoglobin; NDD, newly diagnosed diabetes; NGT, normal glucose tolerance; OGTT, oral glucose tolerance test. 


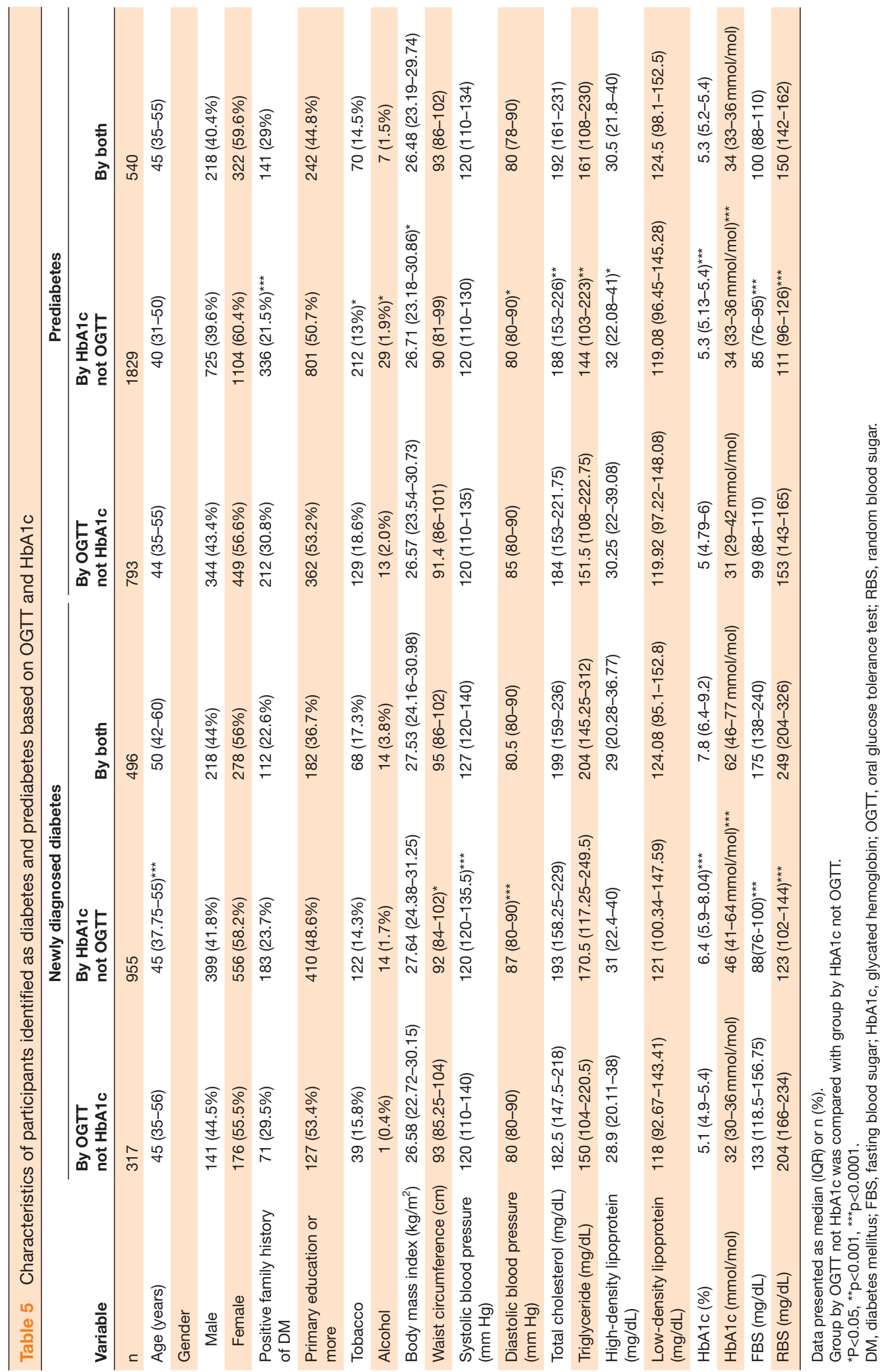


cut-off of $5.8 \%(40 \mathrm{mmol} / \mathrm{mol})$ and $5.7 \%$ (39 mmol/ mol), respectively. ${ }^{27}$ Accordingly, it seems difficult to develop a common HbA1c cut-off worldwide. Gender differences have also been reported in HbAlc levels being higher in men. This study showed no statistically significant difference in genders using both criteria. In a recent study, high specificity and sensitivity of HbAlc versus OGTT was noted after excluding $16 \%$ participants with anemia, but relatively very low number of these participants diagnosed with OGTT were compared with HbA1c, non-representative results. ${ }^{19}$ In this substudy, anemia was not determined as a confounding factor beyond the scope of 2nd NDSP (2016-2017) reported as our limitations. ${ }^{3}$ However, HbAlc is considered as an effective, more convenient and good alternative to FPG to diagnose complications in subjects with known diabetes, better indicator of chronic glycemic levels, has less pre-analytic instability and does not need a fasting or timed sample. Therapeutic decisions are also based on HbA1c value, so to improve the ability for screening prediabetes and NDD, additional FPG or OGTT tests are still necessary. ${ }^{29}$

Based on topical results, considering HbAlc as a diagnostic tool we also have to diagnose anemia first. It is also a debate for new researchers regarding different $\mathrm{HbAlc}$ cut-offs for males and females by WHO and ADA similar to hemoglobin. Therefore, it is necessary to carefully choose HbA1c or FPG (OGTT) as diagnostic criteria.

\section{Strengths and limitations}

An important high point of this substudy is that it is the first study to compare the effect of Pakistani ethnic group on the HbA1c cut-off values for the diagnosis of dysglycemia. HbA1c cut-off values were determined in concordance to OGTT is another strength. We lack data regarding anemia, kidney functions, medication history as confounding factors affecting HbAlc. Apart from few limitations, as subpaper of 2nd NDSP (2016-2017) having community representative data, the findings of this study will contribute to open new horizons of research to use the HbA1c as a diagnostic tool.

\section{CONCLUSION}

The findings from 2nd NDSP demonstrated the disagreement between the findings of OGTT and HbA1c as diagnostic tool for the Pakistani population. As compared with international guidelines, HbAlc threshold for prediabetes and NDD were found to be lower in this part of the world. HbA1c as a diagnostic tool might require ethnic or regional-based modification in cut-off points, validated by relevant community-based epidemiological surveys.

\footnotetext{
Author affiliations

${ }^{1}$ Department of Medicine, Baqai Institute of Diabetology and Endocrinology, Baqai Medical University, Karachi, Pakistan

${ }^{2}$ Department of Biochemistry, Baqai Medical University, Karachi, Pakistan

${ }^{3}$ Department of Research, Baqai Institute of Diabetology and Endocrinology, Baqai Medical University, Karachi, Pakistan
}

${ }^{4}$ Department of Acute Medicine, Whipps Cross University Hospital, Bart's Health NHS Trust, London, UK

${ }^{5}$ Clinical Biochemistry and Psychopharmacology Research Unit, Department of Biochemistry, University of Karachi, Karachi, Pakistan

Acknowledgements The authors would like to thank the Research and Laboratory Department of Baqai Institute of Diabetology and Endocrinology (BIDE), Karachi for data management. The authors would like to thank Abdul Rashid and Muhammad Sohail from Pakistan Health Research Council (PHRC), Karachi. The authors would also like to thank all study subjects for their participation in the second NDSP (2016-2017).

Collaborators NDSP members (with surnames in alphabetical order): Dr Mujeeb Ur Rehman Abro; Dr Khawaja Ishfaq Ahmed; Dr Khurshid Ahmed; Dr Sobia Sabir Ali; Professor Ahmed Bilal; Dr Anam Butt; Professor Bikha Ram Devrajani; ljaz Hayder; Dr Yasir Humayun; Rabia Irshad; Dr Riasat Ali Khan; Dr Asima Khan; Dr Aamir Akram Khowaja; Dr Raheela Khowaja; Professor Qazi Masroor; Dr Maqsood Mehmood; Hassan Moin; Nida Mustafa; Dr Wasif Noor; Dr Huma Qureshi; Mr Ibrar Rafique; Dr Tahir Rasool; Rubina Sabir; Dr M. Arif N. Saqib; Dr Pir Alam Said; Professor Abrar Shaikh; Professor AS Shera; Professor Bilal Bin Younus; Professor Salma Tanveer; Professor Jamal Zafar.

Contributors $\mathrm{AB}$ : concept, design, interpretation of data, edited and approved the final manuscript. AF: concept, design, interpretation of data, edited and approved the final manuscript. KAB: literature search, interpretation of data, wrote the manuscript. NW: literature search, interpretation of data, wrote the manuscript. BT: literature search, interpretation of data, edited and reviewed the final manuscript. IAS: edited and reviewed the final manuscript. NDSP members: members were responsible for the supervision of the survey, concept and design involved in the quality control and data management in their respective areas. All members approved the final submitted version.

Funding The funding source for the study remained same as in the second NDSP (2016-2017). Hemoglobin A1c evaluation and lipid profiles were performed by Pakistan Health Research Council (PHRC). Field visits and data collection including clinical and anthropometric measurements, fasting plasma glucose and 2-hour postglucose load samples were run by the respective teams through their own funds. The whole survey was coordinated and supported by the Baqai Institute of Diabetology \& Endocrinology (BIDE) on their own resources.

Competing interests None declared.

Patient consent for publication Not required.

Ethics approval The study protocol was approved by National Bioethical Committee (NBC) of Pakistan (Ref: No.4-87/17/NBC-226/NBC/2664).

Provenance and peer review Not commissioned; externally peer reviewed.

Data availability statement All data relevant to the study are included in the article. No more additional information available for this study.

Open access This is an open access article distributed in accordance with the Creative Commons Attribution Non Commercial (CC BY-NC 4.0) license, which permits others to distribute, remix, adapt, build upon this work non-commercially, and license their derivative works on different terms, provided the original work is properly cited, appropriate credit is given, any changes made indicated, and the use is non-commercial. See: http://creativecommons.org/licenses/by-nc/4.0/.

ORCID iDs

Abdul Basit http://orcid.org/0000-0002-8041-3360

Asher Fawwad http://orcid.org/0000-0002-2094-9497

\section{REFERENCES}

1 Radhakrishna P, Vinod KV, Sujiv A, et al. Comparison of Hemoglobin $A_{1 c}$ with Fasting and 2-h Plasma Glucose Tests for Diagnosis of Diabetes and Prediabetes among High-risk South Indians. Indian J Endocrinol Metab 2018;22:50-6.

2 Yoon K-H, Lee J-H, Kim J-W, et al. Epidemic obesity and type 2 diabetes in Asia. Lancet 2006;368:1681-8.

3 Basit A, Fawwad A, Qureshi H, et al. Prevalence of diabetes, prediabetes and associated risk factors: second national diabetes survey of Pakistan (NDSP), 2016-2017. BMJ Open 2018;8:e020961.

4 American Diabetes Association. 2. Classification and Diagnosis of Diabetes: Standards of Medical Care in Diabetes-2019. Diabetes Care 2019;42:S13-28. 
5 World Health Organization. Definition and diagnosis of diabetes mellitus and intermediate hyperglycemia: report of a WHO/IDF consultation, 2006.

6 Zhou X, Pang Z, Gao W, et al. Performance of an A1c and fasting capillary blood glucose test for screening newly diagnosed diabetes and pre-diabetes defined by an oral glucose tolerance test in Qingdao, China. Diabetes Care 2010;33:545-50.

7 American Diabetes Association. Diagnosis and classification of diabetes mellitus. Diabetes Care 2014;37:S81-90.

8 Nair M, Prabhakaran D, Narayan KMV, et al. $\mathrm{HbA}(1 \mathrm{c})$ values for defining diabetes and impaired fasting glucose in Asian Indians. Prim Care Diabetes 2011:5:95-102.

9 Herman WH, Cohen RM. Racial and ethnic differences in the relationship between $\mathrm{HbA} 1 \mathrm{c}$ and blood glucose: implications for the diagnosis of diabetes. J Clin Endocrinol Metab 2012;97:1067-72.

10 Nazir A, Papita R, Anbalagan VP, et al. Prevalence of diabetes in Asian Indians based on glycated hemoglobin and fasting and 2-h post-load (75-g) plasma glucose (CURES-120). Diabetes Technol Ther 2012;14:665-8.

11 Selvin E, Crainiceanu CM, Brancati FL, et al. Short-Term variability in measures of glycemia and implications for the classification of diabetes. Arch Intern Med 2007;167:1545-51.

12 Lu ZX, Walker KZ, O'Dea K, et al. A1C for screening and diagnosis of type 2 diabetes in routine clinical practice. Diabetes Care 2010;33:817-9.

13 Kumar PR, Bhansali A, Ravikiran M, et al. Utility of glycated hemoglobin in diagnosing type 2 diabetes mellitus: a communitybased study. J Clin Endocrinol Metab 2010;95:2832-5.

14 Bhowmik B, Diep LM, Munir SB, et al. $\mathrm{HbA}(1 \mathrm{c})$ as a diagnostic too for diabetes and pre-diabetes: the Bangladesh experience. Diabet Med 2013;30:e70-7.

15 Mohan V, Vijayachandrika V, Gokulakrishnan K, et al. A1C cut points to define various glucose intolerance groups in Asian Indians. Diabetes Care 2010;33:515-9.

16 Ho-Pham LT, Nguyen UDT, Tran TX, et al. Discordance in the diagnosis of diabetes: comparison between $\mathrm{HbA} 1 \mathrm{c}$ and fasting plasma glucose. PLoS One 2017;12:e0182192.

17 World Health Organization. Good laboratory practice training manual for the trainer: a tool for training and promoting good laboratory practice (GLP) concepts in disease endemic countries.
2 edn, 2008. http://www.who.int/tdr/publications/documents/glptrainer.pdf

18 American Diabetes Association. Standards of medical care in diabetes. Diabetes Care 2016;39:S13-22.

19 Aamir AH, Ul-Haq Z, Mahar SA, et al. Diabetes prevalence survey of Pakistan (DPS-PAK): prevalence of type 2 diabetes mellitus and prediabetes using $\mathrm{HbA} 1 \mathrm{c}$ : a population-based survey from Pakistan. BMJ Open 2019;9:e025300.

20 Lim W-Y, Ma S, Heng D, et al. Screening for diabetes with HbA1c: test performance of $\mathrm{HbA} 1 \mathrm{c}$ compared to fasting plasma glucose among Chinese, Malay and Indian community residents in Singapore. Sci Rep 2018;8:12419.

21 Cavagnolli G, Comerlato J, Comerlato C, et al. $\mathrm{HbA}(1 \mathrm{c})$ measurement for the diagnosis of diabetes: is it enough? Diabet Med 2011:28:31-5.

22 Nakagami T, Tominaga M, Nishimura R, et al. Is the measurement of glycated hemoglobin A1c alone an efficient screening test for undiagnosed diabetes? Japan national diabetes survey. Diabetes Res Clin Pract 2007;76:251-6.

23 Choi SH, Kim TH, Lim S, et al. Hemoglobin A1c as a diagnostic tool for diabetes screening and new-onset diabetes prediction: a 6-year community-based prospective study. Diabetes Care 2011;34:944-9.

24 Tanaka Y, Atsumi Y, Matsuoka K, et al. Usefulness of stable $\mathrm{HbA}(1 \mathrm{c})$ for supportive marker to diagnose diabetes mellitus in Japanese subjects. Diabetes Res Clin Pract 2001;53:41-5.

25 Bennett CM, Guo M, Dharmage SC. $\mathrm{HbA}(1 \mathrm{c})$ as a screening tool for detection of Type 2 diabetes: a systematic review. Diabet Med 2007;24:333-43.

26 Nida S, Khan DA, ljaz A, et al. Determination of mean glycated haemoglobin in healthy adults of a local population. $J$ Coll Physicians Surg Pak 2017;27:399-403.

27 van 't Riet E, Alssema M, Rijkelijkhuizen JM, et al. Relationship between $\mathrm{A} 1 \mathrm{c}$ and glucose levels in the general Dutch population: the new Hoorn study. Diabetes Care 2010;33:61-6.

28 Rohlfing CL, Little RR, Wiedmeyer HM, et al. Use of GHB (HbA1c) in screening for undiagnosed diabetes in the U.S. population. Diabetes Care 2000;23:187-91.

29 Sherwani SI, Khan HA, Ekhzaimy A, et al. Significance of HbA1c test in diagnosis and prognosis of diabetic patients. Biomark Insights 2016;11:BMI.S38440. 